This document is published in:

Bravo, J. et al. (eds.), 2012. Ambient Assisted Living and Home Care: 4th International Workshop, IWAAL 2012, Vitoria-Gasteiz, Spain, December 3-5, 2012. Proceedings. (Lecture Notes in Computer Science, 7657), Springer, pp.371 -374. DOI: 10.1007/978-3-642-35395-6_50

(C) 2012 Springer-Verlag Berlin Heidelberg 


\title{
EmotionContext: User Emotion Dataset Using Smartphones
}

\author{
Gonzalo Blázquez Gil, Antonio Berlanga, and José M. Molina \\ Applied Artificial Intelligence Group, University Carlos III, Colmenarejo, Spain \\ gbgil@inf.uc3m.es, \{aberlan,molina\}@ia.uc3m.es
}

\begin{abstract}
Mobile device's boom allows to researchers to step forward and design new applications and why not apps which can feel what user feels. This paper presents a smartphone architecture to retrieve user emotions context.
\end{abstract}

\section{Introduction}

Recently, increasing attention has been directed to the study of the human emotional state. Affective Computing (AC) is a branch of AI that deals with the design of systems and devices that can recognize, interpret process and reproduce human affective states. AC term was introduced by Rosalind Picard at MIT in 1997 [3].

$\mathrm{AC}$ is traditionally carried out through video systems [4] or through intrusive systems (wearable sensors) which make difficult to implement in real applications due to user's reluctance to wear devices across their bodies. Smartphones are particularly well-suited to accomplish this task. They are considered a wearable system and also they can operate during long periods of time sensing user activities, routines and the environment.

Moreover, smartphones are changing communication channels computer mediated communication (CMC). Typically, e-mail, social networks (asynchronous) sms, or even instant messaging (synchronous). New communication channels reduce personal contact between speakers to zero. For example, nowadays text messaging and social networks are the most popular way to communicate for teenagers. Taking the advantage of these new smartphone features [1] (Sensors, communication channels and so on), it is possible to create an application which gather user information in order to infer user emotional state.

To conclude this paper presents ContextCare, an architecture to obtain a user labeled emotion information using smartphones and rely on the user social networks posts. Since, these situations to recognize are known a priori, the problem can be tackled as a classification task.

\section{EmotionContext: Architecture and Dataset Proposal}

EmotionContext dataset is developed to satisfy a new wave in smartphones application research community. Nowadays, smartphones are not just a communication tool (telephone or internet), besides, they have embedded sensors which 
may provide countless information about the user. Hence, smartphones can (at least theoretically) hear what you hear, see what you see and even read what you read. This section describes pros and cons to use smartphones sensors in order to obtain user emotion.

\subsection{Emotion Representation}

There exist two different Emotion representation models: Categorical and dimensional. Categorical model of emotion has its roots in the evolutionary theories which claims that emotions are biologically determined, discrete and belong to one of a few groups, these groups are consider fundamental or basic. According with [2] definition of affective state the basic emotions are considered: happiness, sadness, surprise, fear, anger and disgust.

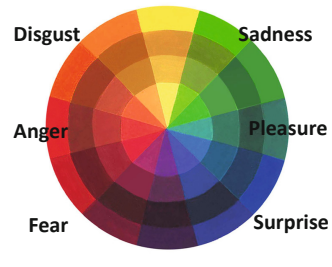

(a)

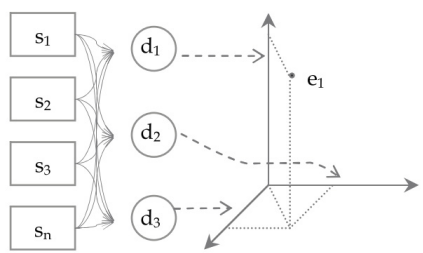

(b)

Fig. 1. Emotion representation: Categorical model (a) and Dimentional model (b)

In contrast to categorical model, dimensional models do not fix a finite set of emotions. Under this model, emotions are described in terms of three components or dimensions [5]. The three dimension approach is synthesized in figure (a) where a concrete emotion (e) is the result of the intersection between every different dimensions $(\mathrm{d})$ whose values are determined by pattern of signals $(\mathrm{s})$.

\subsection{EmotionContext Architecture}

The architecture of our framework is depicted in figure 2. ContextCare is a multimodal architecture which collects sensor information when the user is posting something on internet. The schema shows the three main modules: Smartphone app, SN server and the EmotionContext backend server.

Firstly, SN API's enable developers to access some of the core primitives of each SN including timelines, status updates, pictures, etc. This information is reachable through OAuth authentication protocol which grants access to user information to third-party applications. When users introduce their Facebook or Twitter credentials, they give back an access token, a string denoting a specific scope, lifetime, and other access attributes.

Secondly, EmotionContext smartphone app is developed in Android OS. Sensor module is an Android service which continuously gathers smartphone sensor 


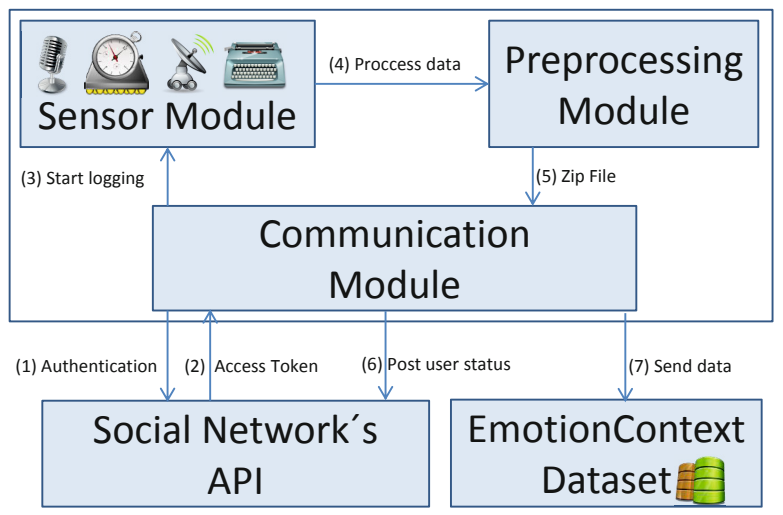

Fig. 2. EmotionContext requests authorization from the SN API using OAuth over HTTP Protocol (1). OAuth Authorization server sends an access token which grants access to user SN protected resources (2). At the same time, Sensor module start to log smartphone data (3). When users post their comment, sensor module sends raw information to the preprocessing module (4) which select the best samples and compress them (5). Finally, Communication Module sends .zip file to EmotionContext dataset via PHP (7).

information according to table 1 . The preprocessing module transforms raw data to a vector features. MEMS data have been stored into a sliding window of 512 samples (approximately 5 seconds), 256 of which overlap with consecutive ones. Besides, the first and last $10 \%$ of windows stored are cropped in order to avoid outliners data, and finally stored in a plain text file.

Finally, EmotionContext Dataset server is a LAMP distribution (Linux, Apache, MySQL and PHP/Python). LAMP distribution support large file size (greater than $2 \mathrm{~GB}$ ), bandwidth throttling to limit the speed of responses in order to not saturate the network and also provides Server-side scripting (PHP) to store every vector features in the MySql database. Figure 3 shows EmotionContext Andorid app screen flow.

Table 1. EmotionContext Dataset Proposal: Matching between smartphone sensor and emotion recognition technique

\begin{tabular}{llll} 
Sensor & API Class & Tecniques & Emotions \\
\hline Front Camera & MediaRecorder & Facial Expression & 6 Basic \& neutral \\
Microphone & MediaRecorder & Emotional Speech & 6 Basic \& neutral \\
Accelerometer & SensorManager & Hand movements & Happy \& anger \\
Location & LocationManager & - & Happiness \& Neutral \\
Typing & Sensing Module & Frequency & Anger \& Neutral \\
SN status & Twitter API & Language Processing & 6 Basic \& neutral \\
\hline
\end{tabular}




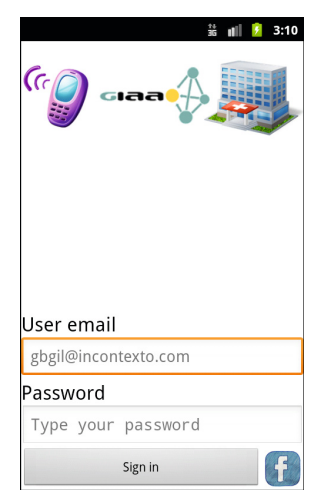

(a)

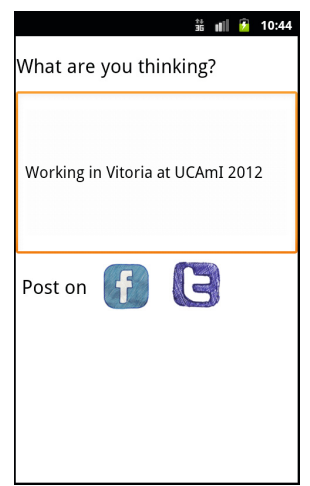

(b)

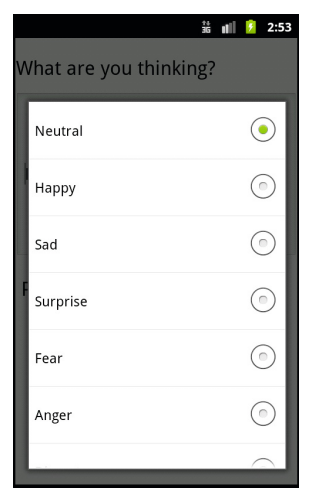

(c)

Fig. 3. Login screen (a), Post user status screen (b) and user emotion chooser (c)

\section{Conclusion}

This paper proposes a smartphone architecture and dataset (EmotionContext) which combines information from a smartphones embedded sensor. EmotionContext dataset is developed to satisfy a new wave, human emotion recognition, in smartphones application research community.

Considering future works are to make a study of the extracted data from the smartphones and of course make an application able to discern the human emotional state. This kind of applications will be very useful in eHealth application, for example monitoring people with mental diseases and sending an alert when the patient is having a disorder.

Acknowledgment. This work was supported in part by Projects CICYT TIN2011-28620-C02-01, CICYT TEC2011-28626-C02-02, CAM CONTEXTS (S2009/TIC-1485) and DPS2008-07029-C02-02.

\section{References}

1. Blazquez Gil, G., Berlanga, A., Molina, J.M.: inContexto: A fusion architecture to obtain mobile context. In: 2011 Proceedings of the 14th International Conference on Information Fusion (FUSION), pp. 1-8. IEEE (2011)

2. Ekman, P., Friesen, W.: Facial action coding system: A technique for the measurement of facial movement (1978)

3. Picard, R.: Affective computing (1997)

4. Reilly, J., Ghent, J., McDonald, J.: Modelling, classification and synthesis of facial expressions. In: Affective Computing: Emotion Modelling, Synthesis and Recognition, pp. 107-132

5. Schlosberg, H.: Three dimensions of emotion. Psychological Review 61(2), 81 (1954) 